\title{
Performance-Based Risk-Sharing Arrangements: U.S. Payer Experience
}

\author{
Joseph A. Goble, PharmD; Brian Ung, PharmD; Sascha van Boemmel-Wegmann, MS, PhD Candidate;
}

Robert P. Navarro, PharmD; and Andrew Parece, MBA

\begin{abstract}
BACKGROUND: As a result of global concern about rising drug costs, many U.S. payers and European agencies such as the National Health Service have partnered with pharmaceutical companies in performance-based risksharing arrangements (PBRSAs) by which manufacturers share financial risk with health care purchasing entities and authorities. However, PBRSAs present many administrative and legal challenges that have minimized successful contract experiences in the United States.
\end{abstract}

OBJECTIVE: To (a) identify drug and disease characteristics and contract components that contribute to successful PBRSA experiences and the primary barriers to PBRSA execution and (b) explore solutions to facilitate contract negotiation and execution.

METHODS: A 37-item, web-based survey instrument (Qualtrics), approximately 20 minutes in duration, was open during July and August 2016. The survey was emailed to 90 pharmacy and medical directors of various health care organizations. Statistical analysis included the Kruskal-Wallis test and chi-square tests to examine differences among payer responses. Survey responses were anonymized and data were aggregated.

RESULTS: Twenty-seven individuals completed the survey ( $30 \%$ completion rate). The majority of respondents worked for regional health plans $(52 \%$, $n=14)$, covering at least 1 million lives $(63 \%, n=17)$, with at least 7 years of managed care experience $(81 \%, n=22)$. A total of 51 PBRSAs were active among respondents at the time of the survey. Easily obtainable and evaluable drug data and medical data were the most important drug and disease attributes for successful PBRSAs, respectively. Pharmacy claims and patient demographic data were assessed as "very easy and inexpensive" to collect. Type and amount of manufacturer payment for drug outcome performance failure, endpoint measurement, and necessary clinical data for drug performance measurement were all critical factors for successful PBRSAs. Standardized contract templates and transparent contract financial risk evaluation and modeling ranked highest among methods of manufacturer facilitation of PBRSAs. This study was limited by sample size and survey questions were limited to explanation of PBRSAs at the disease state level.

CONCLUSIONS: On the basis of PBRSA experiences, respondents noted that drug use in chronic medical conditions and objective drug outcome performance measurements were favorable drug characteristics and serve as the primary source of satisfaction for these types of contracts. Third parties and manufacturers can facilitate the uptake and success of PBRSAs by developing standardized contracting templates in addition to other methods that increase their stake in the arrangement. Looking forward, mounting perceptions of success in this realm of contracting for pharmaceuticals may contribute in the quest for value-based payments in the U.S. health care system.

J Manag Care Spec Pharm. 2017;23(10):1042-52

Copyright $\odot 2017$, Academy of Managed Care Pharmacy. All rights reserved.

\section{What is already known about this subject}

Performance-based risk-sharing arrangements (PBRSAs) for pharmaceuticals are contracts between a health care payer and manufacturer, in which both parties share risk for the performance of a product in a defined patient population, tying payment to outcomes achieved.

While interest regarding PBRSAs in the United States has increased in recent years, much remains unknown about the drug, disease, and contracting elements that lead to perceived success, especially in a climate of many legal and regulatory obstacles.

\section{What this study adds}

This study reports on U.S. health care payer insights into several different PBRSA aspects (e.g., appropriate diseases, required outcomes data, critical contracting points, and methods of facilitation), which have not previously been collectively assessed. Using a web-based survey, 27 U.S. payers provided feedback on 51 active PBRSAs at the time of the survey, as well as on their past and current negotiation experiences.

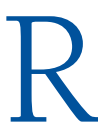

apidly rising health care costs is a critical concern in the United States for public and private payers, health care policymakers, providers, and patients. The gross drug spend in the United States in 2016 rose to $\$ 450$ billion, a 5.8\% increase over 2015, or a per capita spend increase of almost $\$ 1,400{ }^{1}$ Pharmacy program costs increased $21.5 \%$ in 2015 , driven by an increase in utilization and pricing of specialty drugs. In 2015, the pharmacy benefit manager (PBM) Express Scripts, Inc., reported that specialty drugs comprised $27.7 \%$ of its total drug spend and will increase to $50 \%$ by $2018 .{ }^{2}$ When compared with traditional drugs, specialty drugs have shown greater increases in unit pricing $(-2.1 \%$ vs. $11.0 \%)$ and utilization $(1.9 \% \text { vs. } 6.8 \%)^{2}$

U.S. drug prices are generally controlled by market competition, unlike many other nations, including the United Kingdom (UK), whose National Health Service has direct regulatory influence over drug prices and market approvals based on drug value, with similar regulatory oversight in Canada, Australia, and the European Union (EU). ${ }^{3,4}$ However, concerns about rising drug costs exist in these countries despite potential governmental price influences. . $^{5-8}$ 
Performance-Based Risk-Sharing Arrangements: U.S. Payer Experience

TABLE 1 Survey Construction

\begin{tabular}{|c|c|c|}
\hline Domain (Question Number) & Question Types Used & Additional Information \\
\hline Respondent characteristics (1-9) & $\begin{array}{l}\text { Dichotomous, multiple } \\
\text { choice, multiple select }\end{array}$ & $\begin{array}{l}\text { Geographic location, number of years' experience in managed care, organization type, type } \\
\text { and number of covered lives of respondent's current company. }\end{array}$ \\
\hline $\begin{array}{l}\text { Disease areas for past and current } \\
\text { PBRSAs }(10-12,16,21-22)\end{array}$ & $\begin{array}{l}\text { Multiple choice, } \\
\text { multiple select }\end{array}$ & $\begin{array}{l}\text { List of } 23 \text { disease areas included with optional free text response. Current disease areas with } \\
\text { PBRSAs were asked, as well as anticipated and previously pursued disease areas. }\end{array}$ \\
\hline $\begin{array}{l}\text { Drug and disease attributes of } \\
\text { PBRSAs (23) }\end{array}$ & 5-Likert scale & $\begin{array}{l}\text { Respondents rated the importance level of } 19 \text { different PBRSA attributes on a 5-Likert scale } \\
\text { from "critical barrier," low, medium, and high importance to "essential." Inherent and extrinsic } \\
\text { drug attributes (e.g., specialty, novel MOA, low off-label usage) and disease attributes (e.g., top } \\
15 \text { disease by cost or prevalence, acute/chronic disease duration, orphan status) were included. }\end{array}$ \\
\hline Accessibility of data sources (26) & 5-Likert scale & $\begin{array}{l}\text { Respondents rated the ease and cost of } 11 \text { different data types and sources to measure drug } \\
\text { performance (e.g., various types of claims data, electronic medical records) on a 5-Likert scale, } \\
\text { with the lowest category being "very easy and inexpensive to collect" to the highest possible } \\
\text { selection being "impossible or overly expensive to collect." }\end{array}$ \\
\hline $\begin{array}{l}\text { Mechanisms for payment and } \\
\text { reimbursement }(25)\end{array}$ & Multiple select & $\begin{array}{l}\text { Respondents were asked in multiple-select questions their opinions on types of payments } \\
\text { or reimbursements made by pharmaceutical companies that would be included in PBRSAs } \\
\text { such as if they thought the payment type was favorable or unfavorable. Respondents were } \\
\text { also asked if types of reimbursement were included in current, prior, or negotiated but not } \\
\text { executed PBRSAs. }\end{array}$ \\
\hline Critical PBRSA components (24) & $\begin{array}{l}\text { Multiple choice, } \\
\text { discrete adjective scale }\end{array}$ & $\begin{array}{l}\text { Respondents were asked to assess the level of importance of both the payer and the manufac- } \\
\text { turer coming to an agreement on } 11 \text { contract aspects (e.g., drug performance outcome or end- } \\
\text { point to be measured). The contract aspects were rated on a discrete adjective scale comprising } \\
\text { the } 4 \text { choices: "unimportant impact on PBRSA success," "important for PBRSA success," "criti- } \\
\text { cal for PBRSA success," and "uncertain impact on PBRSA success." }\end{array}$ \\
\hline $\begin{array}{l}\text { Payer satisfaction and } \\
\text { dissatisfaction }(13-15,17-20)\end{array}$ & 5-Likert scale & $\begin{array}{l}\text { Satisfaction was rated on a 5-Likert scale with the following options: "very unsatisfied," } \\
\text { "mostly unsatisfied," neither satisfied nor unsatisfied," "mostly satisfied," and "very satisfied." }\end{array}$ \\
\hline $\begin{array}{l}\text { Manufacturer and third-party } \\
\text { facilitation of PBRSAs (32-37) }\end{array}$ & Choice ranking & $\begin{array}{l}\text { Respondents ranked } 8 \text { potential ways manufacturers could assist with making PBRSAs more } \\
\text { workable for payers (e.g., standard contract templates, transparent risk evaluation), with } 1 \\
\text { being the highest rank and } 8 \text { being the lowest. }\end{array}$ \\
\hline PBRSA templates (27-31) & 5-Likert scale & $\begin{array}{l}\text { Respondents were given the definition of PBRSA contract template: structured documents, } \\
\text { preloaded with parameters for important contract attributes specific for each drug and disease. } \\
\text { A } 5 \text {-Likert scale was used to assess whether having such template would have been useful for } \\
\text { their existing PBRSA contracts, and/or for prior or existing contract negotiations, which have } \\
\text { resulted/not resulted in an implemented contract. }\end{array}$ \\
\hline
\end{tabular}

Even with price controls and contracting, the UK and EU health authorities and some U.S. payers have partnered with pharmaceutical companies in performance-based risk-sharing arrangements (PBRSAs), including Aetna and Novartis with sacubitril/valsartan (Entresto), Cigna and Prime Therapeutics and EMD Serono with interferon beta 1-a (Rebif), and Harvard Pilgrim and Amgen with evolocumab (Repatha). ${ }^{9-12}$ PBRSAs help payers mitigate financial risk of new, inexperienced drugs without real-world clinical data and assist manufacturers in achieving patient access and real-world use for newly approved pharmaceutical assets. Since contracts were initiated nearly a decade ago, PBRSAs have proliferated in response to rapidly rising drug prices. ${ }^{13}$ However, PBRSA contracts present many administrative and legal challenges that have minimized successful contract experiences in the United States. ${ }^{14,15}$

The objectives for this U.S. payer primary market research were to (a) identify drug and disease characteristics and contract components that contribute to successful PBRSA experiences and the primary barriers to PBRSA execution, and (b) explore solutions to facilitate contract negotiation and execution.

\section{Methods}

This study invited voluntary participation of U.S. pharmacy and medical directors with experience in relevant drug contracting and formulary decision making from national, regional, and local health insurance plans and PBMs, collectively referred to as "payers," that were involved in pharmacy benefit management or pharmaceutical manufacturer contracting. The invitation email included the study purpose, a description of the survey domains, and a statement of intention for publication of results.

Respondents who self-identified to meet the experience requirement logged into a 37-item, web-based survey instrument (Qualtrics) explicitly developed by the study investigators and 2 Qualtrics-certified experts with the research consultancy Charles River Associates (CRA). The survey incorporated multiple-choice, multiple-select, choice ranking with adjectival scales and 5-point Likert scales, and free text responses to obtain comprehensive insights to payer experiences and opinions related to PBRSAs. The survey included 9 domains (Table 1; the complete survey can be found at the following 
Drug data required easily obtainable/evaluable

Low off-label usage (indications/dosages)

Specialty drug

Drug category with several therapeutic options

No generic or biosimilar expected for $12+$ months

Nonspecialty drug

Drug category with only 1 or 2 drug options

Without PBRSA, drug has prior authorization

Not a biosimilar (original drug only)

Novel MOA

Often used off label (indications/dosages)

Medical data easily obtainable/evaluable

Drug has significant impact on disease therapy

Top 15 disease by drug and/or medical costs

Chronic disease treatment

Top 15 disease by prevalence

Cancer treatment

Orphan disease treatment

Acute disease treatment

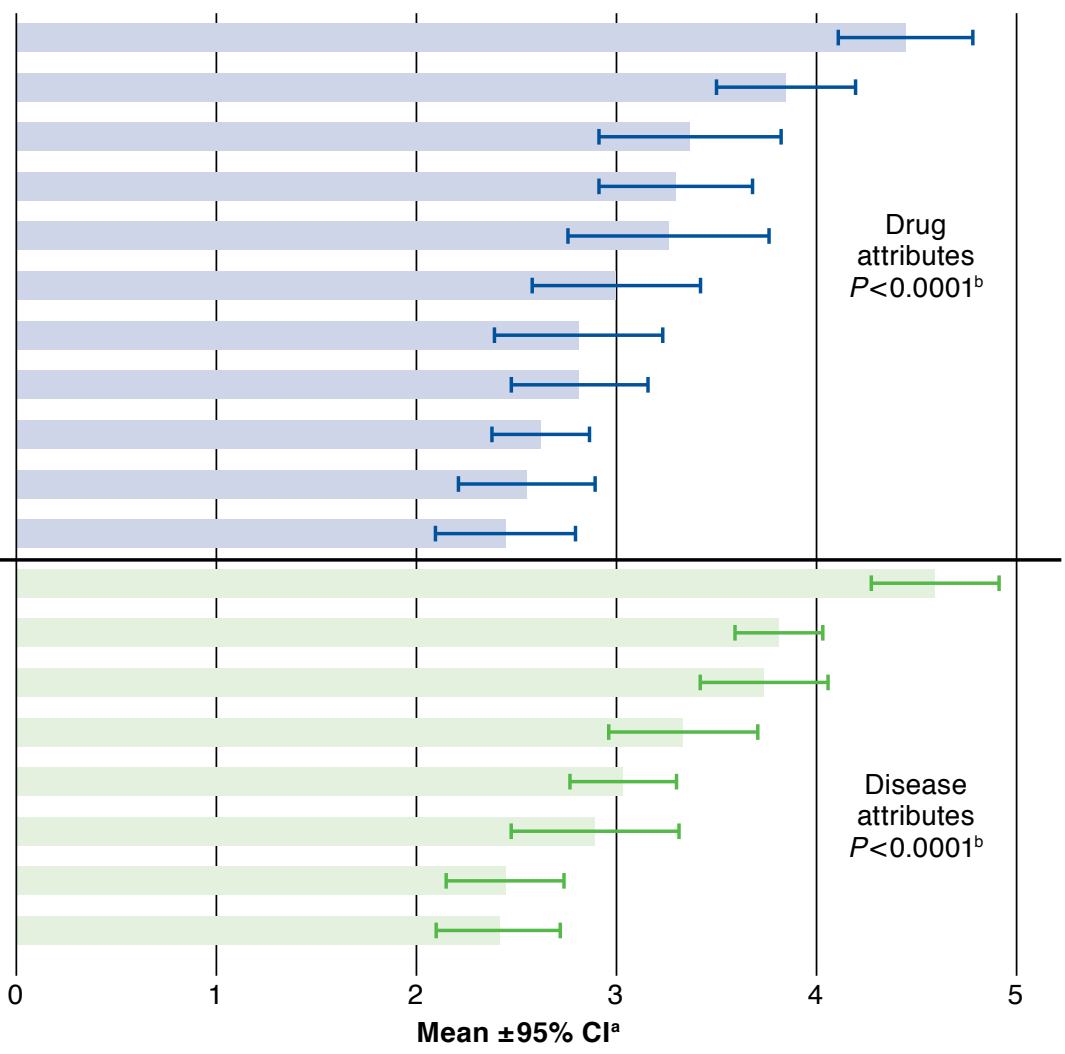

aX-axis: 1 =critical barrier to a PBRSA; $2=$ low importance to PBRSA; $3=$ medium importance to PBRSA; $4=$ high importance to PBRSA; $5=e s s e n t i a l$ to PBRSA

bKruskal-Wallis tests were used to detect differences among responses for level of importance of drug and diseases attributes.

$C I=$ confidence interval $; M O A=$ mechanism of action; $P B R S A=$ performance-based risk-sharing arrangement .

link: https://navarropharmasharefile.com/share?\#/view/s310c5 9d74146788).

Qualified respondents who completed a survey were paid $\$ 150$, with funding provided by CRA. The online survey portal remained open from July through August 2016. Survey responses were de-identified and data were reported at the aggregate level. The study was conducted under a protocol approved by the institutional review board of the University of Florida, Gainesville.

\section{Data Analysis}

The survey responses were coded for descriptive and statistical analysis, and open text responses were collected. Likert scale responses were coded on a continuous scale from 1 to 5 for statistical analysis, and the Kruskal-Wallis test was applied to identify significant differences between attributes compared in the survey (Table 1). Chi-square tests were used to examine differences among categorical responses. Analyses were performed with SAS software package 9.4 (SAS Institute,
Cary, NC) and Microsoft Excel 2016 (Microsoft Corporation, Redmond, WA). All statistical tests were conducted as twosided tests at a significance level of $P<0.05$.

\section{Results}

\section{Respondent Characteristics}

Ninety survey invitations emailed to payer pharmacy and medical directors yielded 32 survey responses (response rate $=35.6 \%$. Five individuals had no experiences with PBRSAs and were disqualified from participating. The remaining 27 individuals completed the survey and were included in the analysis, leading to a completion rate of $30 \%$. Participants were required to provide an answer to every question, but certain questions allowed a "not applicable" or had a "zero or none" option. Incomplete surveys were not included.

All survey respondents $(100 \%, n=27)$ were associated with organizations with commercial, health insurance exchange, and Medicare Part D (Medicare Advantage Prescription Drug plan or prescription drug plan) prescription drug coverage. 
Respondents worked for regional health plans $(52 \%, \mathrm{n}=14)$, integrated delivery systems $(22 \%, \mathrm{n}=6)$, national insurers $(19 \%$, $\mathrm{n}=5)$, and PBMs $(7 \%, \mathrm{n}=2)$. Most organizations covered at least 1 million lives $(63 \%, \mathrm{n}=17)$, with $>100,000$ to 500,000 covered lives being the smallest organization and $>50$ million covered lives being the largest organization represented by the respondents. Most respondents $(81 \%, n=22)$ had been involved with managed care pharmacy program activities for at least 7 years. All survey respondents had involvement with PBRSA contract negotiations with pharmaceutical and/or biotechnology manufacturers, even if not implemented. A majority of respondents $(63 \%, \mathrm{n}=17)$ had experience with the implementation and administration of PBRSAs, including prior and current agreements (Appendix, available in online article).

\section{PBRSA Disease Areas}

Current PBRSAs. The 27 respondents had 51 active PBRSAs. Thirteen respondents had 1 or more existing PBRSAs, with 5 of those having multiple existing PBRSAs, with the most currently active PBRSAs in effect being in cardiovascular heart failure $(n=13)$ and chronic hepatitis $\mathrm{C}$ virus $(\mathrm{HCV} ; \mathrm{n}=8)$. Other disease states with at least 2 PBRSAs included cardiovascular hypertension $(n=5)$, type 2 diabetes mellitus (T2DM; $n=4)$, hyperlipidemia $(n=4)$, oncology $(n=4)$, depression $(n=4)$, osteoporosis $(n=3)$, and rheumatoid arthritis $(R A ; n=2)$. Asthma, chronic obstructive pulmonary disease (COPD), neurology, and multiple sclerosis each had 1 respondent indicate a current PBRSA.

Top Desirable PBRSA Disease Candidates. Respondents stated diseases that displayed desirable PBRSA attributes (e.g., top 15 diseases by drug or medical costs) were hyperlipidemia $(74 \%, \mathrm{n}=20)$, HCV $(67 \%, \mathrm{n}=18)$, T2DM (59\%, $\mathrm{n}=16)$, heart failure $(55 \%, n=15)$, asthma, and COPD (both 44\%, $n=12$ ), and RA $(41 \%, n=11)$. Diseases without desirable PBRSA attributes were irritable bowel syndrome, depression, migraine, diabetic peripheral neuropathy (DPN), and chronic pain.

Top Previously Pursued PBRSA Disease Areas. Respondents reported that previously pursued PBRSAs involved drugs for HCV, hyperlipidemia (both $37 \%, \mathrm{n}=10)$, heart failure $(30 \%$, $n=8$ ), osteoporosis, and oncology (both $26 \%, n=7$ ). Four respondents or fewer had previous PBRSAs with T2DM, RA, asthma, COPD, psoriasis, acute anti-infectives, hypertension, human immunodeficiency virus, gastrointestinal (such as Crohn's or irritable bowel disease), neurological or hematologic conditions, depression, schizophrenia, migraine, DPN, and chronic pain.

\section{Drug and Disease Attributes for PBRSAs}

Drug Attributes. Subjects rated the importance level of different PBRSA attributes (e.g., availability of outcomes data, number of therapeutic options, potential for off-label use) on a 5-point Likert scale with "1" representing the lowest category (critical barrier) and " 5 " representing the highest (essential). The Kruskal-Wallis test showed that the rated importance level was statistically different across the various drug attributes shown in Figure $1(P<0.0001)$. Respondents rated the drug attributes of highest level of importance, for example, required drug data are easily obtainable and evaluable (mean [SD] = 4.44 [1.07]), low offlabel usage (mean $[\mathrm{SD}]=3.85$ [1.08]), specialty drug designation (mean $[\mathrm{SD}]=3.37$ [0.86]), drug category with several therapeutic options (mean $[\mathrm{SD}]=3.30[0.81]$ ), and no generic or biosimilar expected in the next $12+$ months (mean $[S D]=3.26[0.97]$ ).

Drug attributes with lower importance (i.e., means and confidence intervals (CIs) below "medium importance") included drugs with off-label use (mean [SD] = 2.44 [1.07]), and drugs with a novel mechanism of action (mean $[S D]=2.56[0.87]$ ). The means and CIs of the remaining attributes ranged in the "medium importance" level.

Disease Attributes. Similar to drug attribute findings, ratings of the disease attribute importance levels were statistically different (Figure 1, $P<0.0001$ ). The disease attributes of highest level of importance were medical data are easily obtainable/ evaluable (mean $[\mathrm{SD}]=4.59[0.87]$ ), drug is expected to have a significant impact on disease therapy (mean [SD] $=3.81[0.63]$ ), and disease being in the top 15 in drug and/or medical costs (mean $[\mathrm{SD}]=3.74[0.80]$ ). Disease attributes that received lower PBRSA importance ratings (i.e., means and CIs below "medium importance") included acute diseases (mean $[\mathrm{SD}]=2.41$ [0.68]) and orphan diseases (mean $[\mathrm{SD}]=2.44[0.81]$ ). The means and CIs of the remaining items ranged in the "medium importance" level (Figure 1).

\section{Accessibility of Data Sources}

Ratings of the ease and costs for accessing data varied significantly across the respective types of data $(P<0.0001)$. Pharmacy cost and utilization claims (mean $[S D]=1.67$ [0.98]) and patient demographic data (mean $[S D]=2.19[0.77]$ ) were assessed as "very easy and inexpensive" to collect. The types and sources of data rated as being most difficult to obtain were medical chart notes (mean $[S D]=3.85[0.85]$ ) and quality of life and patient-reported outcomes (PROs; mean [SD] = 3.67 [0.72]). The remaining data sources, which included settings where data are captured within electronic medical record (EMR) databases, such as medical laboratory, hospital/emergency department/outpatient and hospital inpatient, ranged in the "moderate ease and cost to collect" level (Figure 2).

\section{Mechanisms for Payment or Reimbursement}

PBRSAs generally require pharmaceutical companies to provide some type of financial reimbursement to a payer if a drug failed to perform as specified in the contract. The payment may be a rebate paid after a contract-defined time period 


\section{FIGURE 2 Ease and Cost of Data Types/Sources to Measure Drug Performance}

\section{5}

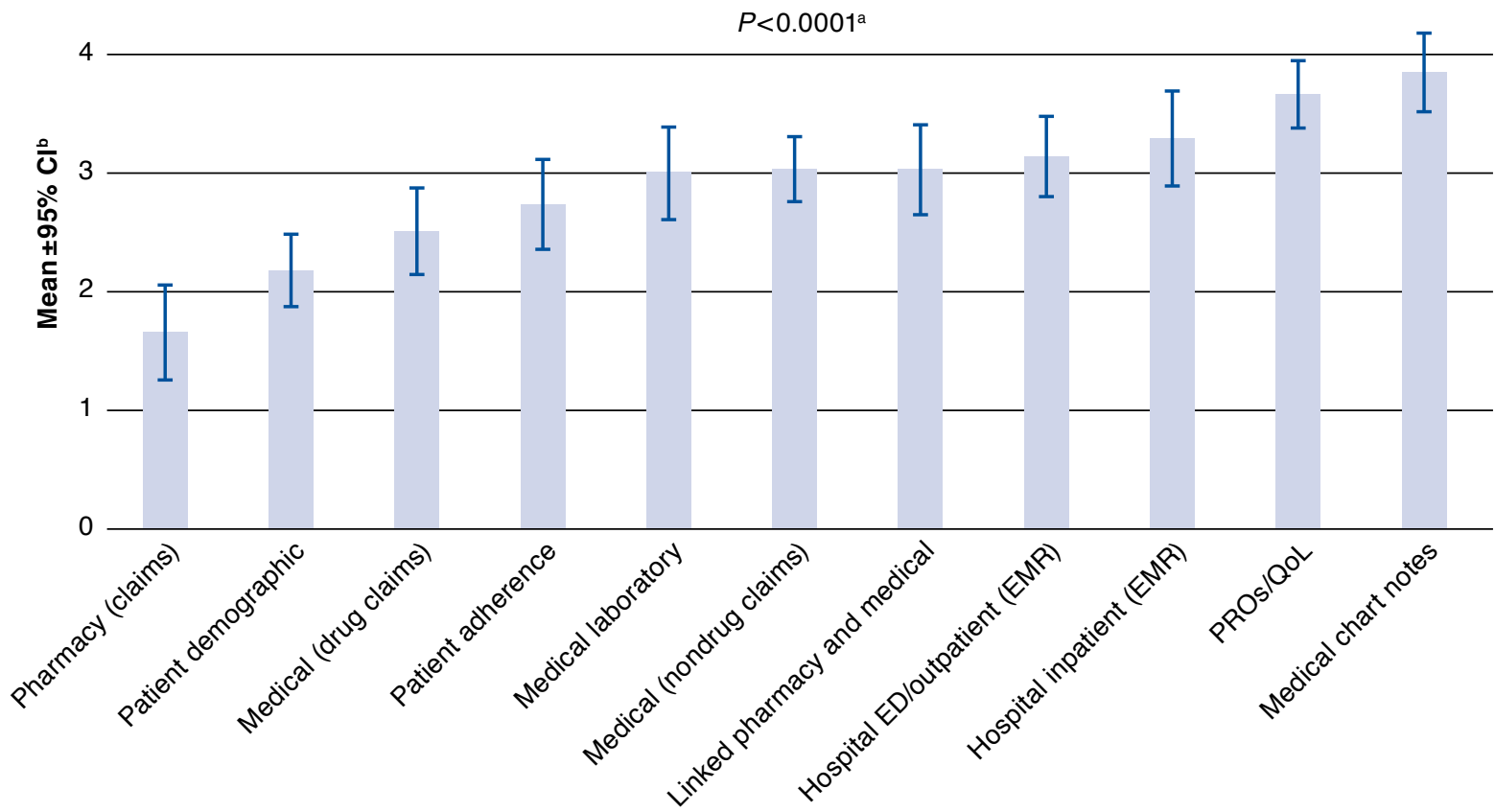

Data Source

aThe Kruskal-Wallis test was used to detect differences among responses for ease and costs of data types/sources to measure drug performance.

bY-axis: 1 =very easy and inexpensive to collect; $2=$ somewhat easy and inexpensive to collect; $3=$ moderate ease and cost to collect; $4=$ difficult and costly to collect; $5=$ impossible or overly expensive to collect.

$C I=$ confidence interval; $E D=$ emergency department; EMR=electronic medical record; PROs = patient-reported outcomes; QoL=quality of life.

(e.g., 6 months) after outcomes data linked to drug performance had been analyzed. One fourth $(25 \%, n=7)$ of the respondents stated that reimbursing the cost of contracted drug failures has been both used in prior PBRSAs and included in current PBRSAs. About half $(48 \%, \mathrm{n}=13)$ of the respondents reported drug cost reimbursement has been in agreements being negotiated but not implemented, and 30\% ( $n=9)$ stated reimbursing drug costs are a favorable reimbursement mechanism for the future. None of the respondents stated that reimbursement for drug costs is inappropriate for PBRSA agreements.

Reimbursement related to direct medical costs associated with drug failure was rated as desirable by respondents, and this type of rebate was included in prior agreements by $19 \%$ $(n=5)$ of respondents, with $15 \%(n=4)$ using this reimbursement in current contracts and 30\% (n=9) of respondents stating that this was part of contracts negotiations that were not implemented. Seventeen respondents (63\%) desired that medical cost reimbursement be included in future PBRSAs. One respondent implied that this form of payment is inappropriate. However, medical cost reimbursement remains primarily at the payer level; patients received financial reimbursement in only $10 \%(n=3)$ of prior agreements. Twenty percent $(n=6)$ of respondents did not implement patient cost-sharing reimbursement for drug or medical costs within their agreements and thought that such reimbursement was inappropriate for PBRSA agreements. Only half $(n=14)$ of the respondents believed reimbursing patients' cost share is desirable for future PBRSAs.

Only 2 respondents indicated that their PBRSAs included reimbursement of costs to treat drug adverse events, while 5 respondents reported this has been in negotiated but not implemented agreements. A majority of respondents $(70 \%$, $\mathrm{n}=19$ ) rated reimbursing drug adverse events as desirable for future PBRSA agreements. Only 1 respondent assessed this type of payment as inappropriate.

Reimbursing costs of alternative drugs required after failure of the contracted drug was uncommon in prior, current, and negotiated (but not implemented) contracts. Seventeen respondents (63\%) regarded reimbursement of alternative drug cost as desirable for future PBRSA agreements, but 4 respondents (15\%) reported this to be inappropriate. 


\section{TABLE 2 Rank Order of Third-Party or Manufacturer Contract Facilitation Methods}

\begin{tabular}{|c|c|c|}
\hline Method of Facilitation & $\begin{array}{c}\text { Payer Ranked }^{\mathrm{a}} \\
(\mathrm{N}=27 ; \text { Mean }[\mathrm{SD}])\end{array}$ & Illustrative Payer Comments \\
\hline $\begin{array}{l}\text { Standardized templates for } \\
\text { contract execution (e.g., by } \\
\text { contract type, TA) }\end{array}$ & $2.92[1.54]$ & $\begin{array}{l}\text { "New area for contracting so setting starting point. [We want to] eliminate confusion and expe- } \\
\text { dite the process." } \\
\text { "Every single deal that fails to get executed after agreement on concept dies during the redline } \\
\text { stage where the legal counsel of both parties cannot live with something. It is an incredible } \\
\text { bottleneck... Speed and momentum are critical." }\end{array}$ \\
\hline $\begin{array}{l}\text { Transparent risk evaluation and } \\
\text { modeling }\end{array}$ & $3.15[2.05]$ & $\begin{array}{l}\text { "Full agreement and understanding by both the payer and pharma upfront potential of contract } \\
\text { and minimization of data confounders." } \\
\text { "Small plans don't have as many resources to validate and replicate the modeling done by the } \\
\text { pharma company to determine the rebate amounts, additional transparency is needed." }\end{array}$ \\
\hline $\begin{array}{l}\text { Full turnkey services for contract } \\
\text { monitoring, administration, and } \\
\text { processing }\end{array}$ & $3.65[1.86]$ & $\begin{array}{l}\text { "Will help facilitate negotiations clinical, medical, legal buy in. Organizations are more apt to } \\
\text { accept when turnkey and others (competitors) have used or are considering." } \\
\text { "Ongoing monitoring is necessary to inform current decision making and to ensure success." }\end{array}$ \\
\hline $\begin{array}{l}\text { Payment by manufacturers for } \\
\text { data or contract }\end{array}$ & $4.19[2.24]$ & $\begin{array}{l}\text { "Reimbursement for plan costs associated with the contract administration." } \\
\text { "This lends itself to NOT being transparent and [consequently] biased. That is the perception } \\
\text { of the MCO's medical, pharmacy, and analytical areas (for those that have one)." }\end{array}$ \\
\hline $\begin{array}{l}\text { Independent third-party } \\
\text { outcomes }\end{array}$ & $4.65[2.11]$ & $\begin{array}{l}\text { "No bias by either party [when independent third parties are used]." } \\
\text { "It is extremely burdensome administratively and the best outcome is the patient has the best } \\
\text { outcomes and likely no dollars earned for all that effort." }\end{array}$ \\
\hline $\begin{array}{l}\text { Partnership with PBRSA } \\
\text { agreements across multiple } \\
\text { disease areas }\end{array}$ & $5.46[2.02]$ & $\begin{array}{l}\text { "... for the disease states that are in the top } 5 \ldots \text { like depression, multiple therapeutic classes of } \\
\text { drugs are used but which drug or drugs produce the highest ROI and improve health-related } \\
\text { outcomes and reduce utilization of health care resources." } \\
\text { "It is hard enough to do one risk arrangement. I don't think I want multiple ones going on at } \\
\text { the same time with the same manufacturer." } \\
\text { "One disease at a time." }\end{array}$ \\
\hline $\begin{array}{l}\text { Assistance with data retrieval and } \\
\text { integration (e.g., pharmacy and } \\
\text { medical records) }\end{array}$ & $5.46[2.19]$ & $\begin{array}{l}\text { "Our plan's legal team will not allow access to data by pharma. An independent third party } \\
\text { MAY be an option." } \\
\text { "We do our own data retrieval." } \\
\text { "Lots of unseen costs involved." }\end{array}$ \\
\hline $\begin{array}{l}\text { Assistance with prescriber } \\
\text { education on PBRSA impact on } \\
\text { formulary selections }\end{array}$ & $6.50[1.62]$ & $\begin{array}{l}\text { "We have a system P\&T that makes formulary decisions and informs our prescribers and we } \\
\text { have a single instance of Epic across all facilities and settings." } \\
\text { "Prescribers belong to many plans and don't care about our contracts." } \\
\text { "If prescribers don't understand the program, the whole process is futile." } \\
\text { "Prescriber education is difficult from pharma since prescribers normally have to deal with } \\
\text { multiple plans and access levels." } \\
\text { "Would like to do this as an MCO and avoid confusion from pharma education." }\end{array}$ \\
\hline
\end{tabular}

apayers were asked to rank all 8 methods of facilitation on a scale of 1-8, with a score of 1 representing the method with the highest potential for PBRSA success and a score of 8 representing the method with the lowest potential.

$M C O=$ managed care organization; $P B R S A=$ performance-based risk-sharing arrangement; $R O I=$ return on investment; $S D=$ standard deviation; $T A=$ therapeutic area .

\section{Critical PBRSA Contract Components}

The majority of participants stated the critical factors for a successful PBRSA included the type and amount of manufacturer payment for drug outcome failure $(93 \%, \mathrm{n}=25)$, the clinical data necessary to measure drug performance $(93 \%, \mathrm{n}=25)$, and availability of cost data to measure drug performance (78\%, $\mathrm{n}=21$ ). All remaining contract aspects were statistically insignificant ( $\chi 2$ goodness-of-fit test $P \geq 0.05$ : observed proportions not different from 50:50) between "important" and "critical" for PBRSA success. None of the listed contract components were evaluated as being unimportant.

\section{Payer Satisfaction and Dissatisfaction}

Satisfaction with Existing PBRSA Agreements. The 27 respondents reported that 51 PBRSA implemented agreements were in effect at the time of survey submission. Payers were "mostly" or "very" satisfied with their current PBRSAs (78\%; $n=40)$, whereas the remaining $22 \%(n=11)$ were reported to be "neither satisfied nor dissatisfied." None of the respondents were unsatisfied with currently implemented PBRSAs for any disease state. Disease areas with "mostly" and "very" satisfied responses were heart failure ( $n=9), \operatorname{HCV}(n=6)$, and hypertension $(n=5)$. Common reasons for satisfaction with current PBRSAs included their being straightforward to define and 
TABLE 3 Key Benefits and Challenges to a PBRSA Contract Template ( $N=27$ )

Illustrative Payer Comments

\begin{tabular}{|c|c|}
\hline \multicolumn{2}{|l|}{ Benefits } \\
\hline \multirow{2}{*}{$\begin{array}{l}\text { Aligns interests of all parties } \\
\text { involved }\end{array}$} & "Keeps both teams on track and levels the field as to what is expected from a final contract." \\
\hline & "Helps legal [from both organizations] get on the same page quickly." \\
\hline \multirow{3}{*}{$\begin{array}{l}\text { Standardizes and expedites } \\
\text { the process }\end{array}$} & "Provides a framework based on past experience that would avoid reinventing the wheel." \\
\hline & $\begin{array}{l}\text { "Each PBRSA agreement from manufacturers is them starting from scratch asking us to come up with something and we } \\
\text { send them back to develop something. We don't have the time." }\end{array}$ \\
\hline & "Standardized approach, no surprises." \\
\hline \multirow{3}{*}{$\begin{array}{l}\text { Creates transparency in } \\
\text { expectations }\end{array}$} & "Keeps both teams on track and levels the field as to what is expected from a final contract." \\
\hline & "Easier to understand, moves agreement, negotiations forward." \\
\hline & "It helps define the parameters on both the business and legal aspects that are approved by the manufacturer for consideration." \\
\hline \multirow{2}{*}{$\begin{array}{l}\text { Provides comprehensive } \\
\text { overview of contract terms }\end{array}$} & "Includes all key elements of contract." \\
\hline & "Ability to use template across multiple contracts-irrespective of product/disease area." \\
\hline \multicolumn{2}{|l|}{ Challenges } \\
\hline \multirow[t]{2}{*}{ Evidence-based approach } & "Need to be based on clinical guidelines that are well accepted or will not move forward." \\
\hline & "[PBRSAs] need real-world validation." \\
\hline \multirow[t]{3}{*}{ Lack of adaptability } & "Not all parts of the template may be needed for each contract, so the template would need to be customizable." \\
\hline & "Would have to be altered dependent upon the disease-state and outcome needed." \\
\hline & $\begin{array}{l}\text { "The MCO or the manufacturer could end up agreeing to a contract that may be not in each other's best interests in terms } \\
\text { of costs and outcomes... PBRSA templates would be with one manufacturer but what about several manufacturers because } \\
\text { using several drugs like asthma and diabetes. That is why we selected HCV as a potential candidate." }\end{array}$ \\
\hline \multirow{2}{*}{$\begin{array}{l}\text { Not widely accepted or } \\
\text { limited applicability }\end{array}$} & "Still not a standard for industry and will require time by pharmaceutical manufacturer and payer to negotiate to their liking." \\
\hline & "May not be applicable to our situation." \\
\hline
\end{tabular}

easily agreed-upon effective performance metrics $(n=27)$, and easy-to-obtain data to measure contract performance $(n=15)$.

Satisfaction with PBRSA Agreements that Are No Longer in Effect. The survey respondents had previous experience with 26 PBRSAs that were no longer in effect at the time of survey. While the majority of respondents $(73 \%, \mathrm{n}=19)$ were indicated to be either "mostly" or "very" satisfied with prior PBRSAs, a portion $(19 \%, n=5)$ reported to be "mostly unsatisfied" with previously implemented PBRSAs. Disease states with unsatisfactory PBRSAs included acute anti-infectives, osteoporosis, hypertension, and heart failure. The most frequent reason cited for PBRSA satisfaction was the straightforward definition of and easily agreed-upon performance metrics $(n=14)$.

The most common reasons for PBRSA dissatisfaction were PBRSAs did not clearly define effective drug performance metrics $(n=4)$ that required drug performance data that were difficult to obtain or measure $(n=4)$ and that involved excessively complex contract negotiations $(n=3)$. Reasons mentioned for executed contracts no longer in effect included entrance of generics into the marketplace $(n=12)$; administrative barriers, such as excessive resources and costs associated with data collection or execution ( $n=9)$; contract expiration $(n=3)$; or other unspecified reasons $(\mathrm{n}=2)$.

\section{Manufacturer and Third-Party Facilitation}

One domain of this survey probed the PBRSA negotiation process. Participants gave opinions on 8 methods to facilitate PBRSA negotiations (e.g., a standardized contract template). A significant difference among the 8 different methods of manufacturer and third-party facilitation was observed $(P<0.0001)$. Standardized contract templates to facilitate execution (mean $[S D]=2.92$ [1.54]) and transparent contract financial risk evaluation and modeling (mean $[\mathrm{SD}]=3.15$ [2.05]) ranked highest among the methods manufacturers could offer to facilitate negotiations. Both approaches were preferred over and statistically different from "assistance with prescriber education on PBRSA impact on formulary selection" (mean $[\mathrm{SD}]=6.50$ [1.62]), "partnership with PBRSA agreements across multiple disease areas" (mean $[\mathrm{SD}]=5.46[2.02]$ ), and "assistance with data retrieval and integration" (mean $[S D]=5.46[2.19]$ ). The complete list of methods for facilitation and corresponding free text commentary can be found in Table 2 .

\section{PBRSA Contract Templates}

Participants stated that contract templates would have been "moderately to very important" to facilitate negotiation in all 3 scenarios: (1) existing PBRSA contracts (mean $[\mathrm{SD}]=3.13$ [1.11]), (2) negotiations that have resulted in an implemented contract (mean $[S D]=3.33[0.90]$ ), and (3) negotiations that 
have not resulted in an implemented contract (mean $[S D]=3.41$ [0.87]). Only survey participants with existing PBRSA contracts $(n=13)$ were required to complete the question in the context of scenario 1. Survey respondents provided free text responses to the key benefits and drawbacks in consideration of a contract template, and major themes were identified from collated responses (Table 3). Payers responded that PBRSA templates would align all party interests, help standardize and expedite the contracting process, create transparency on expectations, and provide a comprehensive overview of necessary contract terms. Conversely, the perceived primary challenges in using such templates for PBRSAs required an evidence-based approach, the need for adaptability (e.g., to accommodate payer, drug, therapeutic classes, novel approaches), and the lack of widespread acceptance due to limited applicability in certain payers.

\section{Discussion}

Successful PBRSAs require selection of the proper drugs and diseases that meet required criteria for importance, financial payment approach, and availability of outcomes measurement data. For past and current PBRSAs, survey participants stated that diseases with available and accepted drug outcome measurement laboratory data (e.g., hyperlipidemia, HCV, and diabetes mellitus) were good candidates for future PBRSAs rather than diseases with subjective outcomes measurements (e.g., pain, depression), with a notable exception being RA, possibly due to the number of drugs in the therapeutic area. Chronic conditions (as opposed to acute conditions), medications with recent drug approvals (where outcomes and financial exposure are unknown without real-world experience), and disease states with several branded treatment options (that offer competition and reasons for the manufacturer to offer contracts) were other characteristics that support the use of PBRSAs. Drugs in disease areas that fit this description may be prime targets for implementing an effective PBRSA.

Reimbursement for drug and medical costs from the payer perspective was frequently cited as a desirable payment mechanism in prior and current PBRSAs. Conversely, patient costs, the cost of adverse events, and costs for switching to alternative drugs were not common payment mechanisms. Survey respondents will also consider novel payment and rebate options within contracts. Over half of the respondents considered the PBRSA payment options offered as desirable, indicating contracting flexibility. As experience with PBRSAs grows, the complexity of payment structures integrated in the agreements may evolve as well. Easily accessible drug outcomes measurement data, agreed to by both parties, is a critical need for successful PBRSAs to minimize resource and cost burdens of contract administration, and the absence of which may present a formidable barrier to contract execution. ${ }^{14,15}$
Respondents were less interested in PBRSAs for acute and orphan diseases compared with chronic diseases and therapeutic areas with multiple drug options. Acute conditions suffer from lack of follow-up and limited financial impact. Orphan diseases have small patient populations and often have limited or only 1 drug option that minimizes manufacturer interest in contracting. As expected, drugs with off-label use potential were undesirables due to inability to measure off-label use or the need for a prior authorization to restrict use.

Easily accessible and inexpensive drug outcomes measurement data are critical for PBRSAs. Pharmacy and medical resource utilization and costs (e.g., claims and encounters data) appear to fit into this category. The wide range of health carerelated data beyond administrative and claims data, such as medical chart record, laboratory, and PRO data, are often not available, incomplete, or expensive to collect. However, these data may be available in integrated delivery systems and those with robust EMRs. Such systems may design payer-specific customization of outcomes data and have reimbursement mechanisms in place for each contracted health care system.

We discovered that payers were collectively more satisfied with current PBRSAs compared with their previous experiences, possibly indicating both parties are learning from past experiences. Reasons for payer satisfaction with past and current performance-based contracts were consistently associated with clear contract terms and definitions and evaluations of performance metrics. Payers did not rate their satisfaction with 8 of the 30 current PBRSAs, which may be reflective of the difficulty in measuring satisfaction as a contract is ongoing. Dissatisfaction seemed to be most common when unable to define and evaluate performance, as well as when negotiations become too complex, highlighting a clear need for succinct negotiation practices that might be better accomplished with more experiences, or standardization of terms in a contract template. Lengthy negotiations, unwillingness to compromise, contract complexity, and the inability to agree on performance metrics and required data can prevent the PBRSA implementation. Trust among both parties is necessary to continue negotiations and seek compromises that do not undermine essential PBRSA components.

Although there is much interest in PBRSAs in recent years, these contracts are relatively uncommon. However, it is likely that they will continue to increase over time as payers attempt to share financial risk with manufacturers for these drugs with unknown effectiveness or financial impact in the real world, and manufacturers attempt to find solutions to obtain formulary access and reimbursement of expensive specialty drugs. A mutual interest to find innovative solutions, and bilateral trust are required to continue to experiment with novel contracts. Validating a definition of success that may be applied universally for PBRSAs will aid in tracking meaningful improvement in the U.S. drug pricing system. ${ }^{16}$ 
PBRSAs are consistent with an overall interest in measurement and reimbursement for quality and high-value drugs. The Center for Medicare \& Medicaid Services' Center for Innovation is experimenting with bundled payments, Quality Payment Programs as part of the Medicare Access and CHIP Reauthorization Act of 2015, and the Oncology Care Model. ${ }^{17}$ PBRSAs provide opportunities to support a valuebased approach to drug assessment and formulary selection and present opportunities for manufacturers to share in financial risk if a drug fails to achieve targeted outcomes.

If PBRSAs are to gain widespread adoption as a method of drug payment for quality outcomes, third parties and manufacturers must offer avenues for collaborative support. ${ }^{16,18}$ Transparent risk evaluation and modeling was 1 process identified in this survey to help accomplish this goal. Manufacturers must demonstrate two-way risk sharing to gain payer trust and ensure that contracts provide mutual benefits for the involved parties.

Payers were moderately interested in a standardized template for contract execution (e.g., by contract type or therapeutic area) to better facilitate negotiations. They recognized the utility of a template in previous and current PBRSAs but also highlighted several potential benefits and challenges (Table 3). Independent third-party outcomes measurement could minimize biases associated with contractual term enforcement, and some organizations are beginning to develop platforms to support this initiative. ${ }^{19}$ Ultimately, improvements in the contracting process must be multifaceted. Identification and characterization of "win-win" scenarios, both in the immediate and long term, should spearhead these improvements. ${ }^{20,21}$

\section{Limitations}

Our main methodological limitation is the small sample size of our surveyed payers. Some with PBRSAs refused to participate because PBRSAs are confidential and present a competitive advantage, and this research had a relatively low representation of integrated delivery systems, large insurers, and PBMs. Also, many payers admitted limited experience with PBRSAs. This research did not explore regulatory and legal hurdles (i.e., Medicaid Best Price, anti-kickback statute) limiting PBRSAs; these concerns have been reported as potential barriers of manufacturer-payer performance contracts. ${ }^{22}$ The structure of the survey questions did not enable respondents to provide feedback on individual experiences in the case of multiple contracts within a given disease. Respondents were also restricted to explaining contracting experiences at disease state level; therefore, experiences surrounding distinct products were not investigated.

Nonetheless, this study represents the most extensive published survey of its kind to date. Additional studies are needed to make stronger conclusions regarding payer experience and perceptions of PBRSAs. Future research in this area could further investigate scenarios and cases where regulatory and legal restrictions prevent adoption of PBRSAs. Taking a closer look at these areas could potentially guide manufacturers to identify optimal stakeholders for contract implementation (e.g., accountable care organizations) as well as determine reasons for less desirable ones, such as in the setting where the patient population is too small to justify negotiation and administrative costs (e.g., small regional health plans).

\section{Conclusions}

Primary research with 27 U.S. respondents had direct experience in 51 PBRSA negotiations and/or implementations. On the basis of past and current PBRSA respondent experiences, respondents noted that drug use in high-cost chronic medical conditions for which drugs have a significant impact on disease management, with easily obtainable objective drug outcome performance measurements (e.g., drug and medical cost and use data), were attributes rated high in importance for PBRSA success. Participants also rated low off-label use and specialty as desirable drug characteristics for PBRSAs. The selection of "specialty drug" suggests a high-cost drug, which is consistent with the payer interest in PBRSAs to help mitigate financial risk of high-cost drugs, especially new drugs with unknown real-world experience.

Third parties and manufacturers can facilitate the uptake and success of PBRSAs by developing standardized but flexible contracting templates with payments related to drug cost and direct medical expense reimbursement related to drug failure. However, studies with more respondents are needed to strengthen the findings and conclusions of this study. Looking forward, concern about rising drug prices and mounting of expectations of innovative contracting for pharmaceuticals may contribute in the quest for value-based drug assessment and reimbursement in the U.S. health care system.

\section{Authors}

JOSEPH A. GOBLE, PharmD, University of Maryland School of Pharmacy, Baltimore; Baylor Scott $E$ White Health, Temple, Texas; and University of Texas, Austin. BRIAN UNG, PharmD, University of Maryland School of Pharmacy, Baltimore, and Rutgers University, Piscataway, New Jersey. ANDREW PARECE, MBA, Charles River Associates, Boston, Massachusetts. SASCHA VAN BOEMMELWEGMANN, MS, PhD Candidate, and ROBERT P. NAVARRO, PharmD, University of Florida, Gainesville.

AUTHOR CORRESPONDENCE: Joseph A. Goble, PharmD, Department of Pharmacy, Baylor Scott \& White Health, 2401 31st St., MS-Ag-407Q, Temple, TX 76508. Tel.: 908.278.4497; Email:Joseph.Goble@BSWHealth.org. 


\section{DISCLOSURES}

The construction of the survey and payment for survey respondents were supported by Charles River Associates. Parece is an employee of Charles River Associates. Goble and Ung are completing fellowship training sponsored by Novartis and Celgene, respectively, but do not have any conflicts of interest and did not receive any funding related to this study. Navarro reports consulting fees from Analysis Group, TEVA, and Amgen, unrelated to this study. Van Boemmel-Wegmann declares no conflict of interest.

Study concept and design were contributed by Navarro, Goble, Ung, and Parece. Navarro took the lead in data collection, along with Goble and Ung, and data interpretation was performed by van Boemmel-Wegmann, Goble, and Ung. The manuscript was written by Goble, Ung, Navarro, and van Boemmel-Wegmann and revised by all of the authors.

\section{REFERENCES}

1. QuintilesIMS Institute. Medicines use and spending in the U.S.: a review of 2016 and outlook to 2021. May 2017. Available at: http://www.imshealth. com/en/thought-leadership/quintilesims-institute/reports/medicines-useand-spending-in-the-us-review-of-2016-outlook-to-2021. Accessed August 28, 2017.

2. Express Scripts. Express Scripts drug trend report 2016. February 2017. Available at: http://lab.express-scripts.com/lab/drug-trend-report. Accessed August 25, 2017.

3. Roos JC, Hyry HI, Cox TM. Orphan drug pricing may warrant a competition law investigation. BMJ. 2010;341:c6471.

4. STAT News. UK patient groups urge government to negotiate drug prices. August 16, 2016. Available at: https://www.statnews.com/pharmalot/2016/08/ 16/drug-prices-cancer-united-kingdom/. Accessed August 25, 2017.

5. BioSpace. Actavis (ACT) UK raised drug prices 12,000 percent, watchdog finds. December 16, 2016. Available at: http://www.biospace.com/news story.aspx?StoryID=442159\&full=1. Accessed August 25, 2017.

6. Childs M. Do drugs really have to be so expensive? BBC News. March 28, 2013. Available at: http://www.bbc.com/news/health-21834442. Accessed August 25, 2017.

7. Gov.UK. CMA fines Pfizer and Flynn $£ 90$ million for drug price hike to NHS. Press release. December 7, 2016. Available at: https://www.gov.uk/government/news/cma-fines-pfizer-and-flynn-90-million-for-drug-price-hiketo-nhs. Accessed August 25, 2017.

8. Reuters. Roche, Bristol-Myers cut cancer drug prices to win UK approval. November 16, 2016. Available at: http://www.reuters.com/article/us-britaincancer-bristolmyers-roche-idUSKBN13C006. Accessed August 25, 2017.

9. Garber AM, McClellan MB. Satisfaction guaranteed-"payment by results" for biologic agents. N Eng J Med. 2007;357(16):1575-77.

10. FierceHealthcare. CIGNA and Merck help customers better manage diabetes. October 28, 2010. Available at: http://www.fiercehealthcare. com/healthcare/cigna-and-merck-help-customers-better-manage-diabetes. Accessed August 25, 2017
11. Fox J, Watrous M. Overcoming challenges of outcomes-based contracting for pharmaceuticals: early lessons from the Genentech-Priority Health pilot. Health Affairs Blog. April 3, 2017. Available at: http://healthaffairs.org/ blog/2017/04/03/overcoming-challenges-of-outcomes-based-contracting-forpharmaceuticals-early-lessons-from-the-genentech-priority-health-pilot/. Accessed August 25, 2017.

12. Amgen. Amgen and Harvard Pilgrim agree to first cardiovascular outcomes-based refund contract for Repatha (evolocumab). News release. May 2, 2017. Available at: https://www.amgen.com/media/news-releases/2017/05/amgen-and-harvard-pilgrim-agree-to-first-cardiovascular-outcomesbased-refund-contract-for-repatha-evolocumab/. Accessed August 25, 2017.

13. White N. Point-of-care assessment of response to high-cost specialty therapy: real-world experience in outcomes-based contracting. AMCP CE session presented at: CBI Formulary, Co-pay, and Access Summit; April 6, 2016; Philadelphia, PA. Available at: http://www.cbinet.com/sites/default/ files/files/White_Nathan_pres_final.pdf. Accessed August 25, 2017.

14. Neumann PJ, Chambers JD, Simon F, Meckley LM. Risk-sharing arrangements that link payment for drugs to health outcomes are proving hard to implement. Health Aff (Millwood). 2011;30(12):2329-37.

15. Garrison LP Jr, Towse A, Briggs A, et al. Performance-based risk-sharing arrangements_-good practices for design, implementation, and evaluation: report of the ISPOR good practices for performance-based risk-sharing arrangements task force. Value Health. 2013;16(5):703-19.

16. AISHealth. Pay-for-value movement turns attention to prescription drugs. Health Business Daily. July 21, 2015. Available at: https://aishealth. com/archive/nhpw071315-04. Accessed August 25, 2017.

17. Centers for Medicare \& Medicaid Services. Oncology care model. Updated July 27, 2017. Available at: https://innovation.cms.gov/initiatives/ oncology-care/. Accessed August 25, 2017.

18. Nayer C, Kish L. Writing the value-based contract. The Health Care Blog. August 6, 2014. Available at: http://thehealthcareblog.com/blog/2014/08/06/ writing-the-value-based-contract/. Accessed August 25, 2017.

19. Inovalon. Inovalon announces agreement with Bristol-Myers Squibb to focus on real world outcomes \& value-based contracting initiatives. Press release. May 19, 2016. Available at: http://www.inovalon.com/press-releases/2016/inovalon-announces-agreement-bristol-myers-squibb-focus-realworld-outcomes. Accessed August 25, 2017.

20. Parece A. Making risk-sharing and clinical performance contracts win-win. Presented at: Next Level Pharma Conference; October 7, 2009; Cambridge, MA

21. Schoonveld E, Kloss S, ZS Associates. Unpacking risk sharing and alternative pricing schemes. Pharm Comm. January/February 2010 online edition. Available at: https://www.zs.com/-/media/files/publications/public/article/ pc-unpacking-risk-sharing.pdf?la=en. Accessed August 25, 2017.

22. Carlson JJ, Garrison LP Jr, Sullivan SD. Paying for outcomes: innovative coverage and reimbursement schemes for pharmaceuticals. J Manag Care Pharm. 2009;15(8):683-87. Available at: http://www.jmcp.org/doi/10.18553/ jmcp.2009.15.8.683. 


\section{APPENDIX Respondent Demographics}

\begin{tabular}{|c|c|c|}
\hline Characteristic & Measure & $\mathrm{N}(\%)$ \\
\hline \multirow[t]{2}{*}{ PBRSA exposure } & Contract negotiations (may or may not have been executed) & $27(100)$ \\
\hline & Implementation and administration & $16 \quad(67)$ \\
\hline \multirow[t]{4}{*}{ Geographic region } & Northeast & $9 \quad(33)$ \\
\hline & Midwest & $6 \quad(22)$ \\
\hline & South & $5 \quad(19)$ \\
\hline & West & $7 \quad(26)$ \\
\hline \multirow[t]{5}{*}{ Experience with managed care pharmacy activities } & $<1$ year & $0 \quad(0)$ \\
\hline & $1-3$ years & 3 (11) \\
\hline & $4-6$ years & $2 \quad(7)$ \\
\hline & $7-9$ years & $3 \quad(11)$ \\
\hline & $10+$ years & $19 \quad(70)$ \\
\hline \multirow[t]{4}{*}{ Organization type } & Local or regional health plan or insurer non-IDS & $14 \quad(52)$ \\
\hline & Multistate ( $>5$ states) or national insurer & $5 \quad(19)$ \\
\hline & IDS & $6 \quad(22)$ \\
\hline & PBM & $2 \quad(7)$ \\
\hline \multirow[t]{7}{*}{ Number of covered lives } & $>100 \mathrm{~K}-500 \mathrm{~K}$ & $3 \quad(11)$ \\
\hline & $>500 \mathrm{~K}-1$ million & $7 \quad(26)$ \\
\hline & $>1$ million-5 million & $10 \quad(37)$ \\
\hline & $>5$ million-10 million & (4) \\
\hline & $>10$ million-25 million & $3 \quad(11)$ \\
\hline & $>25$ million-50 million & $2 \quad(7)$ \\
\hline & $>50$ million & 1 \\
\hline
\end{tabular}

Note: All survey respondents were associated with organizations with commercial (nonexchange), health insurance exchange, Medicare Part D (MA-PD, PDP, or Medicare Advantage) lines of business. All survey respondents reported having direct exposure to, or awareness of, pricing and contracting decisions at their organization as well as experience with implementing PBRSAs.

IDS = integrated delivery system; $M A-P D=$ Medicare Advantage prescription drug plan; $P B M=$ pharmacy benefit manager; PBRSA = performance-based risk-sharing agreement; $P D P=$ prescription drug plan. 